\title{
NEUTRÓFILOS, LEPTINA E CÂNCER: UMA REVISÃO ATUALIZADA DE SUAS RELAÇÕES
}

\author{
NEUTROPHILS, LEPTIN AND CANCER: \\ AN UPDATED REVIEW ABOUT THEIR INTERACTIONS
}

\section{Alice Abend Bardagi ${ }^{1}$, Bruna Calabrese Zanoni ${ }^{1}$, Daniella Moretti Arbore ${ }^{1}$, Débora Oliveira Dias Bicalho', Victória Brandão Barbosa Cera $^{2}$ e Giovanna Rosa Degasperi ${ }^{3}$}

\section{RESUMO}

Os neutrófilos são células abundantes na circulação sanguínea, e constituem a primeira linha de defesa contra patógenos. Possuem a capacidade de migrar para locais de infecção e destruir os microrganismos, através da liberação de seus grânulos tóxicos, produção de espécies reativas de oxigênio (EROs) pela NADPH oxidase, ou, como apontado mais recentemente, pela formação e liberação das chamadas armadilhas extracelulares de neutrófilos (NETs). Muitos estudos têm sido realizados para ampliar a compreensão sobre a formação das NETs e seu papel em algumas doenças, a autoimunidade e o câncer. Alguns estudos mostram que os neutrófilos associados a tumores liberam NETs, no entanto, o seu papel no processo ainda se mantém controverso, podendo representar funções pró ou antitumorais, dependendo do tipo de tumor. A leptina, hormônio produzido pelo tecido adiposo, controla o metabolismo e modula a resposta das células de imunidade inata e adaptativa, dentre elas, os neutrófilos. $\mathrm{O}$ artigo busca explorar os conceitos bioquímicos básicos na formação e sinalização dos neutrófilos e NETs, além de examinar o papel dessas células em tumores e na sua evolução, abordando a possível atuação da leptina neste contexto.

Palavras-chave: NETs, Fagócitos, Tumor.

\section{ABSTRACT}

Neutrophils are a component of the innate immunity and are abundant cells in the blood circulation. They can migrate to infection sites and destroy microorganisms by releasing toxic granules, by producing reactive oxygen species through NADPH oxidase or, more recently discovered, by releasing the neutrophil extracellular traps (NETS). Studies have been made in order to expand the knowledge about its formation and the role it plays in cancer and autoimmune diseases. It has been suggested that neutrophils associated with tumors are likely to release NETs, however the role the NETs play in the tumor process remains unexplained, once it can improve or worsen the immune response. In some cases it can also be associated with autoimmune responses or with tumor cells escape from the initial site. Moreover, studies are trying to link the Leptin, a hormone produced especially by adipose tissue, to the neutrophils' modulation. It is believed that leptin can be associated with poor or high immune response, or even autoimmune response. This article aims to explain basic biochemical concepts in the formation and release of these structures and their role in some diseases such as autoimmunity and cancer, regarding its influence in the disease process, establishing an interface with the probable leptine modulation.

Keywords: NETs, Phagocytes, Tumor.

\footnotetext{
1 Acadêmicas de Medicina - Faculdade de Medicina - Centro de Ciências da Vida - Pontifícia Universidade Católica de Campinas (PUC-Campinas).E-mails: abendalice@gmail.com; bruna.calabresezanoni@yahoo.com.br; dani.arbore@ gmail.com; debibicalho@outlook.com

2 Acadêmica de Medicina - Faculdade de Medicina - Universidade Santo Amaro. E-mail: victoriabrandao@gmail.com 3 Pesquisadora e Docente (PhD) - Faculdade de Medicina - Centro de Ciências da Vida - Pontifícia Universidade Católica de Campinas (PUC-Campinas). E-mail: giovannadegasperi@puc-campinas.edu.br
} 


\section{INTRODUÇÃO}

Os neutrófilos são células constituintes da imunidade inata, especializados no combate a infecções bacterianas e fúngicas, originados da célula-tronco hematopoiética CD34+. O seu estágio de maturação na medula compreende as fases de promielócito, mielócito, metamielócito, neutrófilo banda e neutrófilo segmentado (VON VIETINGHOFF, LEY, 2008). Esse estágio de maturação é dependente de fatores estimuladores das colônias GM-CSF e G-CSF. Durante este processo, diversos receptores são expressos em sua membrana, esses receptores são importantes para o reconhecimento de microrganismos de adesão e de migração (FUTOSI, FODOR, MÓCSAI, 2013). A retenção ou a liberação de neutrófilos pela medula é regulada por receptores de quimiocinas, expressos em sua membrana. O CXCR4 é responsável pela retenção dos neutrófilos na medula e o CXCR2 pela saída dessas células (EASH et al., 2010). Estima-se que em torno de 5-10 x 10 10 neutrófilos sejam formados diariamente (SUMMERS et al., 2010). Os neutrófilos produzidos ficam cerca de 1 - 6 dias na medula antes de serem liberados e, após a liberação, podem ser encontrados por volta de 1 - 2\% na corrente circulatória (SEMERAD et al., 2002), essas células possuem meia-vida em torno de 1.5 - 8 horas (PILLAY et al., 2010).

A partir da corrente circulatória, os neutrófilos podem alcançar sítios de infecção, inflamação ou lesão por quimiotaxia (NOURSHARGH, ALON, 2014). A migração dos neutrófilos para esses sítios é iniciada através da interação com células endoteliais, que expressam moléculas de adesão, a exemplo das selectinas E e P, pelas quais se conectam aos ligantes de glicoproteínas nos neutrófilos, promovendo o seu rolamento pelo endotélio (MCEVER, 2015). Durante o rolamento, o neutrófilo é ativado por intermédio de seus receptores de quimiocinas, o que acarreta a posterior ligação de seus receptores de integrinas às moléculas intracelulares de adesão 1 e 2 (ICAM-1 e ICAM-2), presentes no endotélio (LYCK, ENZMANN, 2015). Essa ligação promove a forte adesão dos neutrófilos ao endotélio, facilitando a diapedese (FILIPPI, 2016), é através da diapedese que ocorre a passagem dos neutrófilos por capilares sanguíneos e vênulas até o local da infecção.

No local da infecção, os neutrófilos atuam contra os microrganismos, por meio da liberação de grânulos, pela produção de espécies reativas de oxigênio (EROs), pela NADPH oxidase, através da liberação de redes, ou por armadilhas extracelulares de neutrófilos (NETs). As NETs são formadas por cromatina e proteínas de origem granular, como a lactoferrina e a mieloperoxidase, e nuclear, a exemplo das histonas. A liberação das NETs pode ocorrer por diversos estímulos fisiológicos, e é dependente de EROs, cuja principal fonte é a NADPH oxidase (COWLAND, BORREGAARD, 2016; ZENG et al., 2019; BURGENER, SCHRODER, 2019).

Após a formação das NETs, pode ou não ocorrer a morte de neutrófilos. A morte de neutrófilos em decorrência desse processo é denominada de netose (REMIJSEN et al., 2011). Classicamente, a captura de neutrófilos em apoptose é mediada por macrófagos presentes nos sítios de infecção, por meio do processo de eferocitose, os macrófagos reconhecem a fosfatidilserina que é expressa por 
neutrófilos em apoptose (GREENLEE-WACKER, 2016). As NETs estão envolvidas com a patogênese de algumas doenças, a exemplo das doenças autoimunes e cânceres (LEE et al., 2017; GARLEY, JABŁOŃSKA, DĄBROWSKA, 2016). Em relação ao câncer, foi observado que em alguns tipos, há a formação dessas estruturas no ambiente tumoral, ou até mesmo na corrente circulatória, entretanto, é controverso o papel das NETs nesse processo.

As funções dos neutrófilos correlacionam-se também com o hormônio leptina, produzido especialmente pelo tecido adiposo (CARON et al., 2018). Suas ações biológicas acontecem através da sua ligação com os seus receptores. Tal interação com esses receptores no hipotálamo desencadeia a via de sinalização Janus Quinase/ Ativadores de Transcrição de Sinal e Transdutores de Ativação (JAK/STAT), relacionada ao controle da fome e saciedade, o que constitui a ação clássica deste hormônio (KWON et al., 2016). Não obstante, receptores de leptina também foram encontrados em células do sistema imune, a exemplo dos neutrófilos, indicando assim, o envolvimento da leptina no controle da resposta imune (NAYLOR, PETRI, 2016). Nos neutrófilos, a leptina é capaz de influenciar a migração e a produção de EROs, contudo, nenhuma correlação entre a leptina e a formação de NETs foi caracterizada, sendo, portanto, um novo campo de estudo a ser investigado e desvendado.

\section{FAGOCITOSE E A PRODUÇÃO DE ESPÉCIES REATIVAS DE OXIGÊNIO}

Os neutrófilos possuem como uma de suas funções centrais, a fagocitose, que promove a morte de patógenos, desde que eles não tenham um mecanismo de resistência à fagocitose e a todos os elementos constituintes deste processo. O processo envolve a captura e engolfamento de microrganismos em um vacúolo, o fagossomo (LEE et al., 2003). O fagossomo é formado após a captura pela extensão dos pseudópodes, a sua formação é mediada por receptores FcyR com o FcyRIIa (CD32), FcyRIIIb (CD16) e FcyRIV, especializados em alvos opsonizados por IgG ou proteínas do sistema complemento. Os alvos opsonizados por IgG são internalizados em menos de 20 segundos (SEGAL et al., 1980). Os neutrófilos, por sua vez, capturam diretamente microrganismos, especialmente através de receptores como os do tipo Toll (TLRs) e a Dectina-1 (ALLEN, ADEREM, 1996; KENNEDY et al., 2007). Os microrganismos capturados são englobados em um fagossomo, estrutura que se une aos grânulos citoplasmáticos dos neutrófilos, os quais favorecem a destruição de patógenos e células infectadas (NIMMERJAHN, RAVETCH, 2006; COLOTTA et al., 1992). Os grânulos são classificados em azurófilos (peroxidase positivo) específicos, ou grânulos de gelatinase (COWLAND, BORREGAARD, 2016).

Além dos grânulos, os neutrófilos também usam o sistema NADPH oxidase de membrana para a produção de EROs contra os patógenos. Este complexo está presente também na membrana do fagossomo, que é formado por quatro proteínas: p47 phox $, \mathrm{p} 67^{\text {phox }}, \mathrm{p} 40^{\text {phox }}, \mathrm{Rac} 2$, e por duas proteínas transmembranas: g91 ${ }^{\text {phox }}$ (NOX2) e p22 $2^{\text {phox }}$ (GROEMPING, RITTINGER, 2005). Entretanto, as proteínas $\mathrm{p} 47^{\text {phox }}, \mathrm{p} 67^{\text {phox }}$ e $\mathrm{p} 40^{\text {phox }}$ formam um complexo localizado no citosol, que mediante estímulo para 
fagocitose, fosforilam Rac2 e p47phox, sendo essa última, fosforilada em múltiplos sítios (NUNES et al., 2013; EL-BENNA et al., 2016). Após a ocorrência das fosforilações, a Rac2 e os complexos p47phox p67phox $^{\text {phe }}$ e p40 phox migram para a membrana simultaneamente, constituindo a NADPH oxidase ativa. (NUNES et al., 2013; EL-BENNA et al., 2016).

O domínio citosólico de gp91 ${ }^{\text {phox }}$ recebe elétrons de NADPH, e os transfere para o oxigênio, produzindo o ânion superóxido $\left(\mathrm{O}_{2}^{-}\right)$. A mieloperoxidase presente nos grânulos forma, a partir do peróxido de hidrogênio $\left(\mathrm{H}_{2} \mathrm{O}_{2}\right)$, o ácido hipocloroso $(\mathrm{HClO})$, que é um composto altamente bactericida. (ROOS et al., 2003; NAUSEEF, 2007; HAMPTON et al., 1998).

Além da fagocitose e da produção de EROs, processos importantes para a destruição de patógenos, os neutrófilos também são capazes de modular respostas imunológicas através da liberação de quimiocinas, a exemplo da CXCL1, CXCL8, CXCL10, CCL2, CCL3 e CCL4, bem como citocinas pró e anti-inflamatórias, incluindo as TNF- $\alpha$, IL-1 $\beta$, IL-6 e TGF- $\beta$, respectivamente (TAMASSIA et al., 2018; NATHAN, 2006; MANTOVANI et al., 2011).

\section{AS ARMADILHAS EXTRACELULARES DOS NEUTRÓFILOS (NETs)}

A liberação de armadilhas extracelulares de neutrófilos (NETs), caracterizada em 2004, é uma estratégia alternativa à fagocitose para destruição de patógenos. A escolha dos neutrófilos por uma das opções é dicotômica e, aparentemente, irreversível. Em algumas situações, quando há defeitos no processo de fagocitose, observa-se um favorecimento da liberação de NETs. No lúpus eritematoso sistêmico, por exemplo, o déficit de fagocitose observado na doença favorece a geração de NETs (BRINKMANN et al., 2004).

Uma hipótese para a escolha dos neutrófilos no que se refere à fagocitose, ou à liberação de NETs, pode estar relacionada à interação destes fagócitos com as plaquetas. As plaquetas, quando ativadas, liberam a proteína HMGB1, que tem a capacidade de indução, não somente da formação de NETs, mas também da inibição da fagocitose, além de favorecer um estado protrombótico em pacientes com câncer (MAUGERI et al., 2014; TADIE et al., 2013; BIANCHI, 2007; FRIGGERI et al., 2010).

Com relação às NETs, dois modelos foram propostos para a sua formação. O primeiro é caracterizado pela liberação de cromatina descondensada e conteúdo granular, ocasionando desintegração da membrana plasmática e posterior netose. O segundo modelo parte do conteúdo nuclear, que é liberado em vesículas para fora da célula, sem gerar danos a membrana celular do neutrófilo (MARTINELLI et al., 2004; ERMERT et al., 2009; YOUSEFI et al., 2009). Essa netose com característica vital, ocorre especialmente em resposta ao Staphylococcus aureus (PILSCZEC et al., 2010). Em ambas as configurações, as NETs são capazes de destruir microrganismos, como bactérias, fungos, protozoários e até mesmo alguns vírus, a exemplo do HIV (BRINKMANN et al., 2004; BRANZK et al., 2014; SAITOH et al., 2012; FUNCHAL et al., 2015; HEMMERS et al., 2011). 
A NADPH oxidase, já discutida anteriormente, além de ser uma forma de indução de morte de microrganismos, por meio da produção de EROs, também está correlacionada com a ativação de proteases granulares intracelulares, e com a geração das NETs. Além da NADPH oxidase, também contribuem para a geração de EROs a mieloperoxidase e a mitocôndria, contudo, a fonte mitocondrial parece não estar envolvida com a geração de NETs (KIRCHNER et al., 2012).

A enzima mieloperoxidase, envolvida com a produção de EROs, assim como a elastase, realiza a descondensação da cromatina. A elastase é liberada dos grânulos azurófilos, migrando para o núcleo onde digere as histonas, promovendo a descondensação da cromatina. A mieloperoxidase atua junto a elastase de forma sinérgica (PAPAYANNOPOULOS et al., 2010). A enzima peptidil arginina deaminase-4 catalisa a citrulinação da histona, favorecendo desta forma a descondensação da cromatina. Estudos mostram que neutrófilos de camundongos knockout para esta enzima não formam NETs (HEMMERS et al., 2011). Pacientes portadores de doença granulomatosa crônica, ou seja, que possuem defeitos no sistema NADPH, também apresentam neutrófilos incapazes de formar redes (FUCHS et al., 2007).

Figura 1 - Formas de atuação dos neutrófilos contra microrganismos: Degranulação, Fagocitose, NETs.

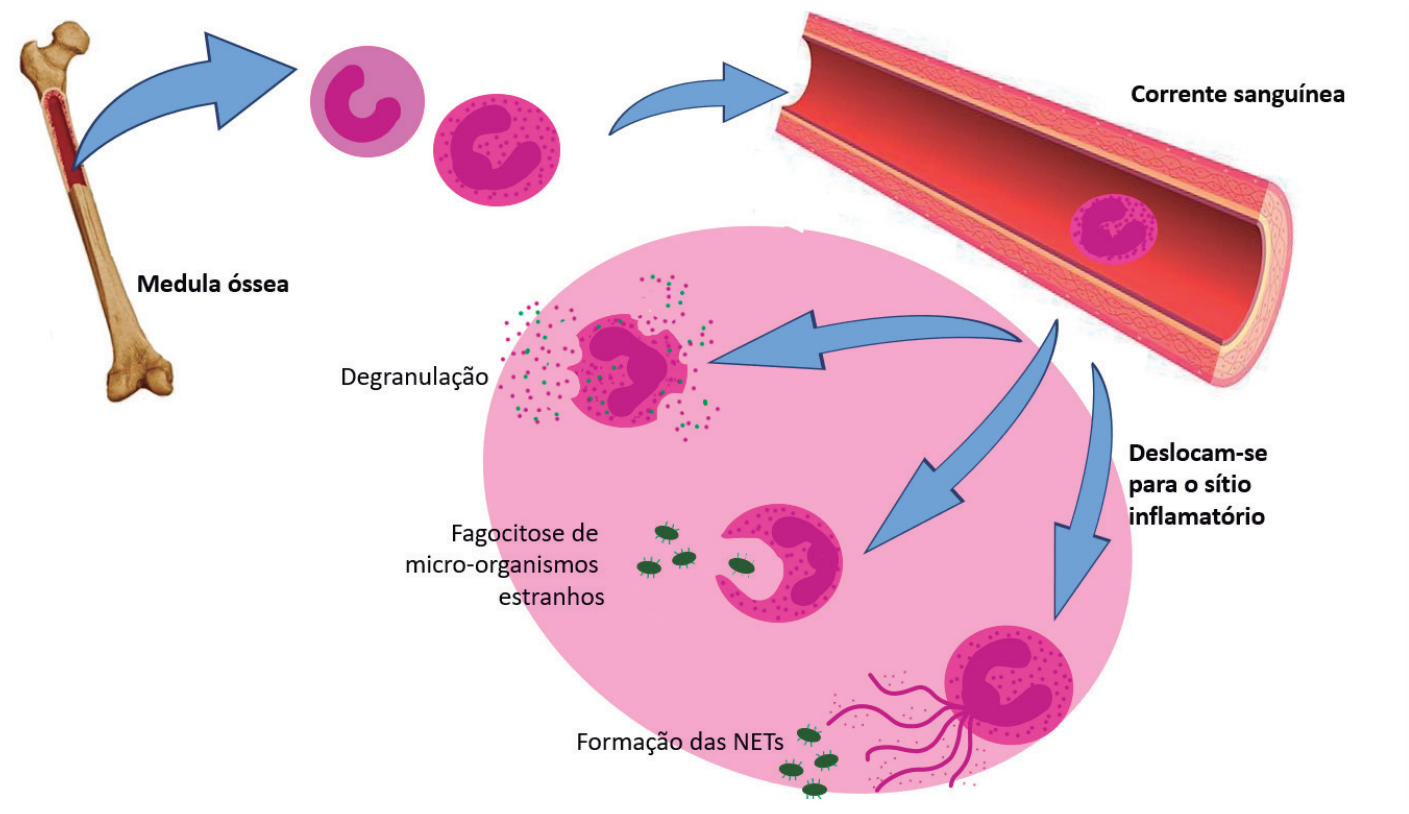

Fonte: Elaborado pelos autores.

\section{LEPTINA E NEUTRÓFILOS}

A leptina é um hormônio peptídico de $16 \mathrm{KDa}$ codificado pelo gene Lep ou db. A leptina exerce suas ações biológicas, por meio da ligação ao seu receptor (LEPR ou ObRb), o qual pertence à família dos receptores de classe I de citocinas. Existem várias isoformas do receptor (OBRa-OBRf), sendo a OBRb, aquela a possuir um domínio citoplasmático mais longo, e a que parece ser, a única isoforma 
capaz de transduzir o sinal da leptina (TARTAGLIA, 1997). A leptina é produzida principalmente pelo tecido adiposo. A sua ligação à forma longa do receptor OBRb hipotalâmico induz ativação de JAK2, que fosforila a porção intracelular do receptor, dando início a uma via de sinalização que regula o balanço energético e a saciedade. Entretanto, na obesidade, essa sinalização é disfuncional. Tal ausência de resposta à leptina, circulante no hipotálamo, é conhecida como resistência à leptina (SÁINZ et al., 2015). Sabe-se que indivíduos obesos apresentam frequentemente essa resistência, o que leva a um bloqueio dos sinais hipotalâmicos que favorecem a saciedade, além do acúmulo de leptina circulante (SÁINZ et al., 2015). Esse acúmulo pode induzir a proliferação de células tumorais que tenham os receptores para leptina (HAN, WANG, 2015; LIPSEY et al., 2016; SZYSZKA et al., 2018).

Diversas evidências indicam que a leptina é capaz de modular o sistema imunológico, atuando diretamente nos leucócitos (MATARESE et al., 2005). Com relação aos neutrófilos, esses expressam a forma curta $\mathrm{ObRa}$ do receptor, a qual parece ser suficiente para desencadear sinalização mediada pela leptina (ZARKESH-ESFAHANI et al., 2004; BRUNO et al., 2005). Essa sinalização leva a um bloqueio da morte celular de neutrófilos humanos in vitro, alterando a produção de EROs, retardando a clivagem de proteínas pró-apoptóticas da mitocôndria e a liberação de citocromo c (BRUNO et al., 2005). Com relação aos EROs, uma redução da sua liberação foi observada em neutrófilos incubados in vitro e em fluido sinovial em concentrações aumentadas de leptina (GAJEWSKI et al., 2015). A leptina também favoreceu a migração de neutrófilos, porém, ela foi dependente da produção da citocina TNF- $\alpha$ por monócitos e da expressão da quimiocina CXCL1 (SOUZA et al., 2018; ZARKESH-ESFAHANI et al., 2001; ZARKESH-ESFAHANI et al., 2004). Ainda em relação à migração, também foi demonstrado que a mutação no receptor de leptina Q223R, prejudicou a migração de neutrófilos para o cólon durante a infecção por Entamoeba histolytica, o que pode explicar a diarreia e a mortalidade observadas em crianças infectadas por esse parasita (NAYLOR et al., 2014). Em estudo recente (JOSE et al., 2018), foi observado que polimorfismos no receptor de leptina, levaram a um aumento da expressão do receptor de quimiocina CXCR2 e à liberação de neutrófilos da medula, em resposta a infecção por Clostridium difficile.

Alterações em concentrações de leptina circulante parecem estar envolvidas com a função e capacidade migratória de neutrófilos. Em modelo experimental animal, o tratamento com dieta hiperlipídica, que acarreta aumento da leptina circulante, foi capaz de induzir a um aumento da população de neutrófilos, os quais apresentaram maior capacidade de migração e de produção de EROs, especialmente ânion superóxido (DO CARMO et al., 2013; BROTFAIN et al., 2015), entretanto, em humanos, concentrações fisiológicas de leptina não afetam as suas funções (KAMP et al., 2013).

A leptina também está envolvida com a sepse e outras doenças inflamatórias. Diversos estudos demonstram um aumento da concentração plasmática desse hormônio nesta condição infecciosa, indicando o seu possível envolvimento com a resposta imune que ocorre na sepse (BEHNES et al., 2012). Observou-se a formação de NETs na sepse como um modo de controle da infecção, porém, 
parece não existir correlação entre NETs e o hormônio leptina nesse processo (LIPINSKA-GEDIGA et al., 2017).

Figura 2 - Leptina na atuação e funções de neutrófilo.

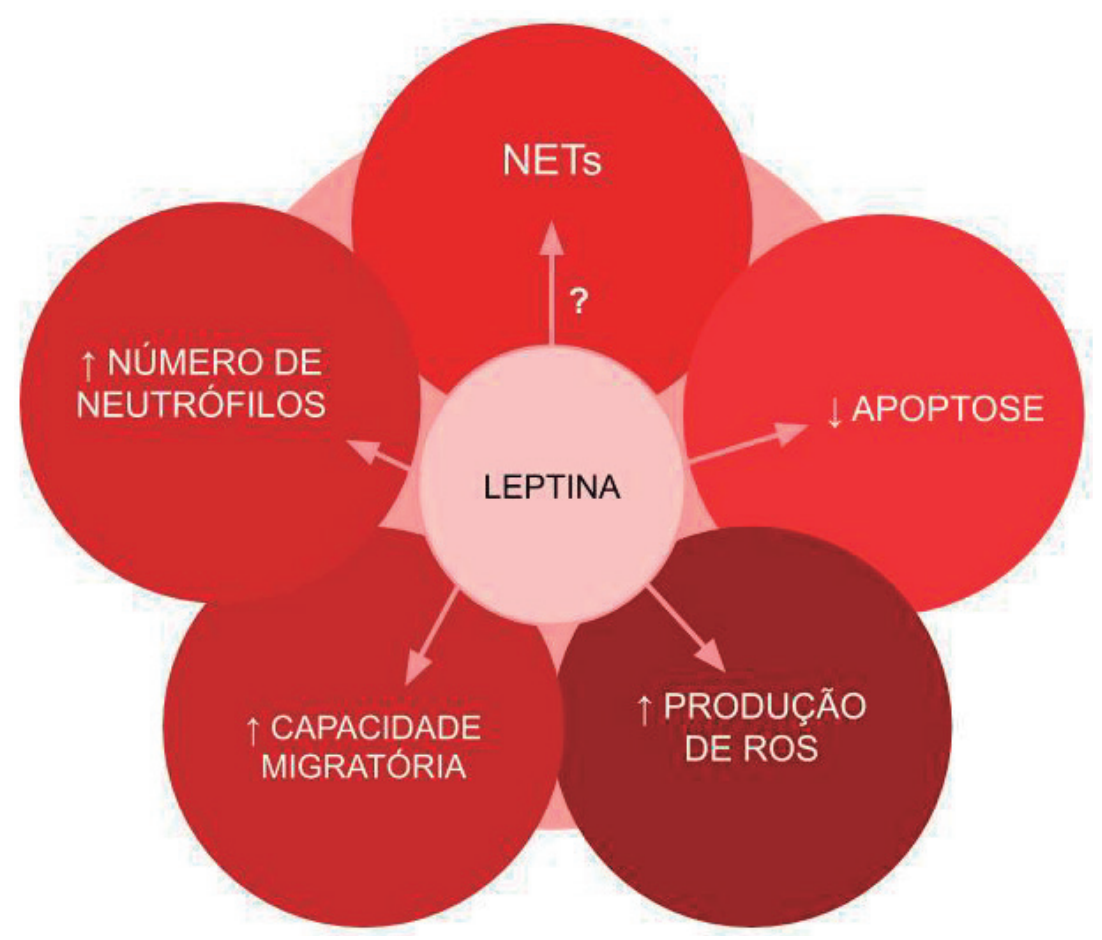

Fonte: Elaborado pelos autores.

\section{NEUTRÓFILOS, NETS E CÂNCER}

Neutrófilos N1 (neutrófilo antitumoral) e N2 (neutrófilo pró-tumoral) foram caracterizados em ambientes tumorais (COFFELT et al., 2015). Os estados de polarização dos neutrófilos, associados ao tumor, são dependentes de fatores do microambiente tumoral, entretanto, o papel destas células nesse contexto ainda é controverso.

A presença de neutrófilos foi detectada em diversos tumores sólidos, como nos tumores de cabeça e pescoço, carcinoma hepatocelular, carcinoma de células renais, entre outros. Nestes estudos, os neutrófilos correlacionam-se com prognóstico ruim para os indivíduos portadores desses tumores (JENSEN et al., 2009; LI et al., 2011; TRELLAKIS et al., 2011; POWELL, HUTTENLOCHER, 2016).

Com relação aos tumores de mama, foi demonstrado que a inibição do receptor de quimiocina CXCR2, importante para a migração de neutrófilos, foi capaz de melhorar a eficácia da quimioterapia (ACHARYYA et al., 2012; SHARMA et al., 2013; XU et al., 2018; CHAN et al., 2016). Neste sentido, a inibição de receptores de citocinas pode ser uma estratégia clínica contra a migração dos neutrófilos para o ambiente tumoral (BERTINI et al., 2004).

No câncer de pulmão, os neutrófilos foram capazes de ativar a resposta imunológica, através da estimulação da resposta das células $\mathrm{T}$, todavia, estas células foram inibidas por neutrófilos em 
ambiente de ascites de tumores ovarianos (ERUSLANOV et al., 2014; SINGEL et al., 2016). De tal forma que, por meio do relato dos estudos apontados, observamos ações dos neutrófilos que podem favorecer ou prejudicar o desenvolvimento tumoral.

Alguns estudos mostram que neutrófilos associados a tumores liberam NETs, porém, enquanto em alguns deles as NETs exercem um papel antitumoral, por induzir morte de células tumorais ou ativar a imunidade antitumoral, em outros, as NETs parecem de alguma forma desfavorecer a resposta imune tumoral, ou até mesmo promover o escape de células tumorais (DEMERS et al., 2016; GARLEY et al., 2016).

É evidente, quando comparados aos dos grupos controles saudáveis, os níveis de NETs no plasma de pacientes com câncer de pulmão, adenocarcinoma de pâncreas e câncer de bexiga foram substancialmente superiores (OKLU et al., 2017). Da mesma forma, de acordo com esses achados, a liberação de NETs em pacientes com câncer colorretal também é significativamente mais alta, em comparação aos controles saudáveis. No entanto, não foram observadas diferenças com relação à liberação de NETs entre pacientes portadores de câncer de pulmão, e controles de pacientes saudáveis (ARPINATI et al., 2020).

Na leucemia linfóide crônica (LLC), foi observado que, os pacientes portadores da doença apresentam alta concentração de neutrófilos circulantes (MANUKYAN et al., 2017), com maior propensão para liberação de suas NETs (PODAZA et al., 2017). Essa maior propensão está relacionada aos altos níveis de IL-8 (MILLRUD et al., 2017; PODAZA, RISNIK, 2019). Curiosamente, foi sugerido que uma possível explicação para a alta frequência de infecções por bactérias encapsuladas, observada em pacientes com LLC, deve-se a possíveis falhas no mecanismo de liberação de NETs pelos neutrófilos. (MORRISON et al., 2014; BEITER et al., 2006).

Estudos recentes mostraram que, em um modelo experimental animal para leucemia, a cromatina liberada no sangue através da geração de NETs, funciona como fator pró coagulante e protrombótico (MARTINOD, WAGNER, 2014; DEMERS et al., 2012). A inibição das NETs, nesse contexto, pode funcionar como uma potencial terapia para a trombose associada ao câncer, considerando que essa condição vascular é uma causa importante de morte em pacientes portadores de neoplasia.

\section{CONCLUSÃO}

Os neutrófilos são células constituintes da imunidade inata, especializadas no combate a infecções bacterianas e fúngicas. Entre os seus mecanismos de defesa imunológica está a formação das NETs. Os neutrófilos possuem receptores específicos para leptina, a qual sabidamente modula a produção de EROs e a degranulação neutrofílica, entretanto, não se tem descrição a respeito desse hormônio no processo de formação das NETs. 
As NETs estão envolvidas com a patogênese de algumas doenças, como as autoimunes e cânceres. Com relação ao câncer, neutrófilos N1 (neutrófilo antitumoral) e N2 (neutrófilo pró-tumoral) foram caracterizados em ambientes tumorais, e a sua polarização tem sido associada a fatores do microambiente tumoral, contudo, o papel destas células neste contexto ainda é controverso. Os neutrófilos N1 auxiliam nesse processo, por induzir a morte de células tumorais, ou ativar a imunidade antitumoral, em outros casos, porém, as NETs parecem de alguma forma desfavorecer a resposta imune tumoral, ou até mesmo promover o escape de células tumorais.

Por fim, ainda há muito a ser pesquisado acerca das NETs, seus fatores de modulação e influência em patologias, em especial ao câncer. A compreensão desses mecanismos pode auxiliar na condução e no tratamento de muitas doenças, como as que foram relacionadas, e impactar na melhora de prognóstico.

\section{REFERÊNCIAS}

ACHARYYA, S. et al. A CXCL1 paracrine network links cancer chemoresistance and metastasis. Cell, v. 150, n. 1, p. 165-178, 2012.

ALLEN, L.A.; ADEREM, A. Molecular definition of distinct cytoskeletal structures involved in complement-and Fc receptor-mediated phagocytosis in macrophages. The Journal of Experimental Medicine, v. 184, n. 2, p. 627-637, 1996.

ARPINATI, L. et al. NETosis in cancer: a critical analysis of the impact of cancer on neutrophil extracellular trap (NET) release in lung cancer patients vs. mice. Cancer Immunology Immunotherapy, v. 69, n. 2, p. 199-213, 2020.

BEHNES, M. et al. Alterations of leptin in the course of inflammation and severe sepsis. BMC Infectious Diseases, v. 12, n. 1, p. 1-11, 2012.

BEITER, K. et al. An endonuclease allows Streptococcus pneumoniae to escape from neutrophil extracellular traps. Current Biology, v. 6, n. 4, p. 401-407, 2006.

BERTINI, R. et al. Noncompetitive allosteric inhibitors of the inflammatory chemokine receptors CXCR1 and CXCR2: prevention of reperfusion injury. Proceedings of the National Academy of Sciences, v. 101, n. 32, p. 11791-11796, 2004. 
BIANCHI, M.E. DAMPs, PAMPs and alarmins: all we need to know about danger. Journal of Leukocyte Biology, v. 81, n. 1, p. 1-5, 2007.

BRANZK, N. et al. Neutrophils sense microbe size and selectively release neutrophil extracellular traps in response to large pathogens. Nature Immunology, v. 15, n. 11, p. 1017, 2014.

BRINKMANN, V. et al. Neutrophil extracellular traps kill bacteria. Science, v. 303, n. 5663, p. 1532$1535,2004$.

BROTFAIN, E. et al. Neutrophil functions in morbidly obese subjects. Clinical \& Experimental Immunology, v. 181, n. 1, p. 156-163, 2015.

BRUNO, A. et al. Apoptotic pathways are inhibited by leptin receptor activation in neutrophils. The Journal of Immunology, v. 174, n. 12, p. 8090-8096, 2005.

BURGENER, S.S.; SCHRODER, K. Neutrophil extracellular traps in host defense. Cold Spring Harbor Perspectives in Biology, v. 12, n. 7, p. a037028, 2020.

CARON, A. et al. Leptin and brain-adipose crosstalks. Nature Reviews Neuroscience, v. 19, n. 3, p. $153,2018$.

CHAN, T.S. et al. Metronomic chemotherapy prevents therapy-induced stromal activation and induction of tumor-initiating cells. Journal of Experimental Medicine, v. 213, n. 13, p. 2967-2988, 2016.

COFFELT, S.B. et al. IL-17-producing $\gamma \delta \mathrm{T}$ cells and neutrophils conspire to promote breast cancer metastasis. Nature, v. 522, n. 7556, p. 345-348, 2015.

COLOTTA, F. et al. Modulation of granulocyte survival and programmed cell death by cytokines and bacterial products. Blood, v. 80, n. 8, p. 2012-2020, 1992.

COWLAND, J.B.; BORREGAARD, N. Granulopoiesis and granules of human neutrophils. Immunological Reviews, v. 273, n. 1, p. 11-28, 2016.

DEMERS, M. et al. Cancers predispose neutrophils to release extracellular DNA traps that contribute to cancer-associated thrombosis. Proceedings of the National Academy of Sciences, v. 109, n. 32, p. 13076-13081, 2012. 
DEMERS, M. et al. Priming of neutrophils toward NETosis promotes tumor growth. Oncoimmunology, v. 5, n. 5, p. e1134073, 2016.

DO CARMO, L.S. et al. A high-fat diet increases interleukin-3 and granulocyte colony-stimulating factor production by bone marrow cells and triggers bone marrow hyperplasia and neutrophilia in Wistar rats. Experimental Biology and Medicine, v. 238, n. 4, p. 375-384, 2013.

EASH, K.J. et al. CXCR2 and CXCR4 antagonistically regulate neutrophil trafficking from murine bone marrow. The Journal of Clinical Investigation, v. 120, n.7, p. 2423-2431, 2010.

EL-BENNA J. et al. Priming of the neutrophil respiratory burst: role in host defense and inflammation. Immunological Reviews, v. 273, n. 1, p. 180-193, 2016.

ERMERT, D. et al. Mouse neutrophil extracellular traps in microbial infections. Journal of Innate Immunity, v. 1, n. 3, p. 181-193, 2009.

ERUSLANOV, E.B. et al. Tumor-associated neutrophils stimulate T cell responses in early-stage human lung cancer. The Journal of Clinical Investigation, v. 124, n. 12, p. 5466-5480, 2014.

FILIPPI, M.D. Mechanism of diapedesis: importance of the transcellular route. Advances in Immunology, v. 129 , p. 25-33, 2016.

FRIGGERI, A. et al. HMGB1 inhibits macrophage activity in efferocytosis through binding to the avß3-integrin. American Journal of Physiology-Cell Physiology, v. 299, n. 6, p. C1267-C1276, 2010.

FUNCHAL, G.A. et al. Respiratory syncytial virus fusion protein promotes TLR-4-dependent neutrophil extracellular trap formation by human neutrophils. PloS ONE, v. 10, n. 4, p. e0124082, 2015.

FUNCHS, T.A. et al. Novel cell death program leads to neutrophil extracellular traps. The Journal of Cell Biology, v. 176, n. 2, p. 231-241, 2007

FUTOSI, K.; FODOR, S.; MÓCSAI, A. Reprint of Neutrophil cell surface receptors and their intracellular signal transduction pathways. International Immunopharmacology, v. 17, n. 4, p. 1185-1197, 2013.

GAJEWSKI, M. et al. The effect of leptin on the respiratory burst of human neutrophils cultured in synovial fluid. Reumatologia, v. 53, n. 1, p. 21, 2015. 
GARLEY, M.; JABLONSKA, E.; DABROWSKA, D. NETs in cancer. Tumor Biology, v. 37, n. 11, p. 14355-14361, 2016.

GREENLEE-WACKER, M.C. Clearance of apoptotic neutrophils and resolution of inflammation. Immunological Reviews, v. 273, n. 1, p. 357-370, 2016.

GROEMPING, Y.; RITTINGER, K. Activation and assembly of the NADPH oxidase: a structural perspective. Biochemical Journal, v. 386, n. 3, p. 401-416, 2005.

HAMPTON, M.B.; KETTLE, A.J.; WINTERBOURN, C.C. Inside the neutrophil phagosome: oxidants, myeloperoxidase, and bacterial killing. Blood, The Journal of the American Society of Hematology, v. 92, n. 9, p. 3007-3017, 1998.

HAN, T.J.; WANG, X. Leptin and its receptor in hematologic malignancies. International Journal of Clinical and Experimental Medicine, v. 8, n. 11, p. 19840, 2015.

HEMMERS, S. et al. PAD4-mediated neutrophil extracellular trap formation is not required for immunity against influenza infection. PLoS ONE, v. 6, n.7, p. e22043, 2011.

JENSEN, H.K. et al. Presence of intratumoral neutrophils is an independent prognostic factor in localized renal cell carcinoma. Journal of Clinical Oncology, v. 27, n. 28, p. 4709-4717, 2009.

JOSE, S. et al. Leptin receptor q223r polymorphism influences neutrophil mobilization after Clostridium difficile infection. Mucosal immunology, v. 11, n. 3, p. 947-957, 2018.

KAMP, V.M. et al. Physiological concentrations of leptin do not affect human neutrophils. PLoS ONE, v. 8, n. 9, p. e73170, 2013.

KENNEDY, A.D. et al. Dectin-1 promotes fungicidal activity of human neutrophils. European Journal of Immunology, v. 37, n. 2, p. 467-478, 2007.

KIRCHNER, T. et al. The impact of various reactive oxygen species on the formation of neutrophil extracellular traps. Mediators of Inflammation, v. 2012, p. 849136, 2012.

KWON, O.; KIM, K.W.; KIM, M.S. Leptin signalling pathways in hypothalamic neurons. Cellular and Molecular Life Sciences, v. 73, n. 7, p. 1457-1477, 2016. 
LEE, K.H. et al. Neutrophil extracellular traps (NETs) in autoimmune diseases: a comprehensive review. Autoimmunity Reviews, v. 16, n. 11, p. 1160-1173, 2017.

LEE, W.L.; HARRISON, R.E.; GRINSTEIN, S. Phagocytosis by neutrophils. Microbes and Infection, v. 5, n. 14, p. 1299-1306, 2003.

LI, Y.W. et al. Intratumoral neutrophils: a poor prognostic factor for hepatocellular carcinoma following resection. Journal of Hepatology, v. 54, n. 3, p. 497-505, 2011.

LIPINSKA-GEDIGA, M. Neutrophils, NETs, NETosis-old or new factors in sepsis and septic shock? Anaesthesiology Intensive Therapy, v. 49, n. 3, p. 235-240, 2017.

LIPSEY, C.C. et al. Oncogenic role of leptin and Notch interleukin-1 leptin crosstalk outcome in cancer. World Journal of Methodology, v. 6, n. 1, p. 43, 2016.

LYCK, R.; ENZMANN, G. The physiological roles of ICAM-1 and ICAM-2 in neutrophil migration into tissues. Current Opinion in Hematology, v. 22, n. 1, p. 53-59, 2015.

MANTOVANI, A. et al. Neutrophils in the activation and regulation of innate and adaptive immunity. Nature Reviews Immunology, v. 11, n. 8, p. 519-531, 2011.

MANUKYAN, G. et al. Neutrophils in chronic lymphocytic leukemia are permanently activated and have functional defects. Oncotarget, v. 8, n. 49, p. 84889, 2017.

MARTINELLI, S. et al. Induction of genes mediating interferon-dependent extracellular trap formation during neutrophil differentiation. Journal of Biological Chemistry, v. 279, n. 42, p. 44123-44132, 2004.

MARTINOD, K.; WAGNER, D.D. Thrombosis: tangled up in NETs. Blood, v. 123, n. 18, p. 2768-2776, 2014.

MATARESE, G.; MOSCHOS, S.; MANTZOROS, C.S. Leptin in immunology. The Journal of Immunology, v. 174, n. 6, p. 3137-3142, 2005.

MAUGERI, N. et al. Activated platelets present high mobility group box 1 to neutrophils, inducing autophagy and promoting the extrusion of neutrophil extracellular traps. Journal of Thrombosis and Haemostasis, v. 12, n. 12, p. 2074-2088, 2014. 
MCEVER, R.P. Selectins: initiators of leucocyte adhesion and signalling at the vascular wall. Cardiovascular Research, v. 107, n. 3, p. 331-339, 2015.

MILLRUD, C.R. et al. NET-producing CD16high CD62Ldim neutrophils migrate to tumor sites and predict improved survival in patients with HNSCC. International Journal of Cancer, v. 140, n. 11, p. 2557-2567, 2017.

MORRISON, V.A. Infections in patients with leukemia and lymphoma. Infectious Complications in Cancer Patients, v. 161, p. 319-349, 2014.

NATHAN, C. Neutrophils and immunity: challenges and opportunities. Nature Reviews Immunology, v. 6, n. 3, p. 173-183, 2006.

NAUSEEF, W.M. How human neutrophils kill and degrade microbes: an integrated view. Immunological Reviews, v. 219, n. 1, p. 88-102, 2007.

NAYLOR, C. et al. Leptin receptor mutation results in defective neutrophil recruitment to the colon during Entamoeba histolytica infection. MBio, v. 5, n. 6, p. e02046-14, 2014.

NAYLOR, C.; PETRI JR, W.A. Leptin regulation of immune responses. Trends in Molecular Medicine, v. 22, n. 2, p. 88-98, 2016.

NIMMERJAHN, F.; RAVETCH, J.V. Fc $\gamma$ receptors: old friends and new family members. Immunity, v. 24, n. 1, p. 19-28, 2006.

NOURSHARGH, S.; ALON, R. Leukocyte migration into inflamed tissues. Immunity, v. 41, n. 5, p. 694-707, 2014.

NUNES, P.; DEMAUREX, N.; DINAUER, M.C. Regulation of the NADPH oxidase and associated ion fluxes during phagocytosis. Traffic, v. 14, n. 11, p. 1118-1131, 2013.

OKLU, R. et al. Neutrophil extracellular traps are increased in cancer patients but does not associate with venous thrombosis. Cardiovascular Diagnosis and Therapy, v. 7, p. S140, 2017. Suplemento 3.

PAPAYANNOPOULOS, V. et al. Neutrophil elastase and myeloperoxidase regulate the formation of neutrophil extracellular traps. Journal of Cell Biology, v. 191, n. 3, p. 677-691, 2010. 
PILLAY, J. et al. In vivo labeling with $2 \mathrm{H} 2 \mathrm{O}$ reveals a human neutrophil lifespan of 5.4 days. Blood, The Journal of the American Society of Hematology, v. 116, n. 4, p. 625-627, 2010.

PILSCZEK, F.H. et al. A novel mechanism of rapid nuclear neutrophil extracellular trap formation in response to Staphylococcus aureus. The Journal of Immunology, v. 185, n. 12, p. 7413-7425, 2010.

PODAZA, E. et al. Neutrophils from chronic lymphocytic leukemia patients exhibit an increased capacity to release extracellular traps (NETs). Cancer Immunology Immunotherapy, v. 66, n. 1, p. 77-89, 2017.

PODAZA, E.A.; RISNIK, D.M. Neglected players: Tumor associated neutrophils involvement in chronic lymphocytic leukemia progression. Oncotarget, v. 10, n. 20, p. 1862-1863, 2019.

POWELL, D.R.; HUTTENLOCHER, A. Neutrophils in the tumor microenvironment. Trends in Immunology, v. 37, n. 1, p. 41-52, 2016.

REMIJSEN, Q. et al. Dying for a cause: NETosis, mechanisms behind an antimicrobial cell death modality. Cell Death \& Differentiation, v. 18, n. 4, p. 581-588, 2011.

ROOS, D.; VAN BRUGGER, R.; MEISCHL, C. Oxidative killing of microbes by neutrophils. Microbes and Infection, v. 5, n. 14, p. 1307-1315, 2003.

SÁINZ, N. et al. Leptin signaling as a therapeutic target of obesity. Expert Opinion on Therapeutic Targets, v. 19, n. 7, p. 893-909, 2015.

SAITOH, T. et al. Neutrophil extracellular traps mediate a host defense response to human immunodeficiency virus-1. Cell Host \& Microbe, v. 12, n. 1, p. 109-116, 2012.

SEGAL, A.W.; JONES, O.T. Absence of cytochrome b reduction in stimulated neutrophils from both female and male patients with chronic granulomatous disease. FEBS Letters, v. 110, n. 1, p. 111-114, 1980.

SEMERAD, C.L. et al. G-CSF is an essential regulator of neutrophil trafficking from the bone marrow to the blood. Immunity, v. 17, n. 4, p. 413-423, 2002. 
SHARMA, B. et al. Targeting CXCR2 enhances chemotherapeutic response, inhibits mammary tumor growth, angiogenesis, and lung metastasis. Molecular Cancer Therapeutics, v. 12, n. 5, p. 799-808, 2013.

SINGEL, K.L. et al. Ovarian cancer ascites-activated neutrophils suppress T cell proliferation in a contact-dependent mechanism. Journal of Immunology, v. 196, n. 1, p. 211.16, 2016.

SOUZA-ALMEIDA, G. et al. Leptin mediates in vivo neutrophil migration: involvement of tumor necrosis factor-alpha and CXCL1. Frontiers in Immunology, v. 9, p. 111, 2018.

SUMMERS, C. et al. Neutrophil kinetics in health and disease. Trends in Immunology, v. 31, n. 8, p. 318-324, 2010.

SZYSZKA, M. et al. Effects of leptin on leptin receptor isoform expression and proliferative activity in human normal prostate and prostate cancer cell lines. Oncology Reports, v. 39, n. 1, p. 182-192, 2018.

TADIE, J.M. et al. HMGB1 promotes neutrophil extracellular trap formation through interactions with Toll-like receptor 4. American Journal of Physiology-Lung Cellular and Molecular Physiology, v. 304, n. 5, p. L342-L349, 2013.

TAMASSIA, N. et al. Cytokine production by human neutrophils: Revisiting the "dark side of the moon”. European Journal of Clinical Investigation, v. 48, n. S2, p. e12952, 2018.

TARTAGLIA, L.A. The leptin receptor. Journal of Biological Chemistry, v. 272, n. 10, p. 60936096, 1997.

TRELLAKIS, S. et al. Polymorphonuclear granulocytes in human head and neck cancer: enhanced inflammatory activity, modulation by cancer cells and expansion in advanced disease. International Journal of Cancer, v. 129, n. 9, p. 2183-2193, 2011.

VON VIETINGHOFF, S.; LEY, K. Homeostatic regulation of blood neutrophil counts. The Journal of Immunology, v. 181, n. 8, p. 5183-5188, 2008.

$\mathrm{XU}, \mathrm{H}$. et al. CXCR2 promotes breast cancer metastasis and chemoresistance via suppression of AKT1 and activation of COX2. Cancer Letters, v. 412, p. 69-80, 2018. 
YOUSEFI, S. et al. Viable neutrophils release mitochondrial DNA to form neutrophil extracellular traps. Cell Death \& Differentiation, v. 16, n. 11, p. 1438-1444, 2009.

ZARKESH-ESFAHANI, H. et al. High-dose leptin activates human leukocytes via receptor expression on monocytes. The Journal of Immunology, v. 167, n. 8, p. 4593-4599, 2001.

ZARKESH-ESFAHANI, H. et al. Leptin indirectly activates human neutrophils via induction of TNF- $\alpha$. The Journal of Immunology, v. 172, n. 3, p. 1809-1814, 2004.

ZENG, M.Y. et al. The roles of NADPH oxidase in modulating neutrophil effector responses. Molecular Oral Microbiology, v. 34, n. 2, p. 27-38, 2019. 
